\title{
THE DECOMPOSITION OF MEROMORPHIC FUNCTIONS INTO RATIONAL FUNCTIONS OF UNIVALENT FUNCTIONS
}

BY

\author{
LYNN H. LOOMIS(1)
}

1. Introduction. If $f(z)$ is meromorphic at a point $z_{0}$ then it is an elementary fact that the multivalent character of $f(z)$ in the neighborhood of $z_{0}$ is like that of a power. By this we mean that for some integer $n$, the function $\left[f(z)-f\left(z_{0}\right)\right]^{1 / n}$ (or $f(z)^{-1 / n}$ if $z_{0}$ is a pole; for simplicity we assume $f(z)$ to be regular at $\left.z_{0}\right)$ is univalent in a sufficiently small circle $\left|z-z_{0}\right|<r$. This property is clearly a local property. Let us consider other ways of expressing it. We may say that the map of a sufficiently small circle $\left|z-z_{0}\right|<r$ under $w=f(z)$ is a Riemann configuration $\left({ }^{2}\right)$ which can be imbedded in the Riemann surface of $\left[w-f\left(z_{0}\right)\right]^{1 / n}$. Or we may say that $f(z)$ has a decomposition $f(z) \equiv f_{2}\left(f_{1}(z)\right)$, where $\zeta=f_{1}(z)$ is univalent in a sufficiently small circle $\left|z-z_{0}\right|<r$, and $f_{2}(\zeta)$ is the rational function $\zeta^{n}+f\left(z_{0}\right)$.

It is the object of this paper to study the analogue in the large of the local property just described. That is, given $f(z)$ meromorphic in a simplyconnected region $R$, we shall investigate the decomposition $f(z) \equiv f_{2}\left(f_{1}(z)\right)$, where $f_{2}(\zeta)$ is a rational function, and $\zeta=f_{1}(z)$ is univalent in $R$. In particular we shall answer the following questions. If $f(z)$ is meromorphic in $|z|<1$, what are necessary and sufficient conditions that $f(z)$ be decomposable as above? What modifications of these conditions are equivalent to restricting $\zeta=f_{1}(z)$ to mapping functions for special classes of simply-connected regions, say Jordan regions? What modifications are equivalent to restricting $f_{2}(\zeta)$ to special classes of rational functions, say polynomials?

2. Survey. In this section we shall attempt to motivate the line of development pursued and indicate the nature of the theorems to be proved, as well as introduce whatever terminology will be needed and state what facts will be assumed.

The general question which we have raised is this: given $f(z)$ meromorphic in a simply-connected region $R$ of the $z$-plane, what conditions are necessary and sufficient that $f(z)$ can be decomposed, $f(z) \equiv f_{2}\left(f_{1}(z)\right)$, where $f_{2}(\zeta)$ is a rational function, and $\zeta=f_{1}(z)$ is univalent in $R$ ? It is evidently sufficient to discuss the case where $R$ is the unit circle $|z|<1$. For if $R$ has only one boundary

Presented to the Society, October 26, 1940; received by the editors June 25, 1940.

(1) Society of Fellows, Harvard University.

(2) The term Riemann surface is of ten taken to refer to the complete Riemann surface of a given analytic function. The term Riemann configuration is used to include both Riemann surfaces in the above sense and pieces of Riemann surfaces. 
point, or is the extended $z$-plane, then $f_{1}(z)$ must be linear and $f(z)$ must itself be a rational function. Excluding this trivial case, $R$ has at least two boundary points and can be mapped onto the unit circle $|z|<1$. And the combination of the mapping function with $f_{1}(z)$ gives another univalent function.

Now let us consider the properties of a function which has such a decomposition. In order to do this we shall need a notion of neighborhood for boundary points. By a half-neighborhood of a point $z_{0}$ on the boundary $|z|=1$ we shall mean the intersection of the unit circle $|z|<1$ with a circle $\left|z-z_{0}\right|<r$. Note that a half-neighborhood of $z_{0}$ does not contain $z_{0}$. By a rational surface we shall mean the Riemann surface of the inverse of a rational function. Now if $f(z)$ is decomposable, it is trivially true that for every $z_{0}$ on $|z|=1$ there is a half-neighborhood whose map under $w=f(z)$ is a Riemann configuration which can be imbedded in a rational surface. In our fundamental (and most general) decomposition theorem, proved in $\$ 3$, this necessary condition will be proved to be sufficient.

Along the circumference, however, the multivalent character of $f(z)$ is not in general of the simple type occurring at interior points, where $f(z)$ is like a power $\zeta^{n}+c$. Consider for example a plane simply-connected region in the $\zeta$-plane, of which not all boundary points are attainable. Then if $\zeta=f_{1}(z)$ maps the unit circle $|z|<1$ onto this region, there is at least one point $z_{0}$ on $|z|=1$ where $f_{1}(z)$ has more than one limiting value. Let $w=f_{2}(\zeta)$ be a rational function having zeros of $f_{2}^{\prime}(\zeta)$ at two such limiting values. Then $f_{2}\left(f_{1}(z)\right)$ is a decomposable function, but its multivalent character at $z_{0}$ is not like that of a power. We shall say that $f(z)$ is rationally-multivalent at $z_{0}$ if there is a neighborhood (half-neighborhood if $z_{0}$ lies on the boundary) of $z_{0}$ which $w=f(z)$ maps onto a Riemann configuration which can be imbedded in a rational surface (i.e., if there is a rational function $w=f_{2}(\zeta)$ such that $f_{2}^{-1}(f(z))$ is meromorphic and univalent in a sufficiently small neighborhood of $z_{0}$ ). If the Riemann surface of an $n$th root is sufficient for this imbedding (as it is, for instance, at all points of $|z|<1$ ) we shall say that $f(z)$ is power-multivalent at $z_{0}$.

Now let us consider the decomposition of $f(z)$ with respect to each of the two functions into which $f(z)$ is decomposed. The function $\zeta=f_{1}(z)$ is univalent on $|z|<1$ and is therefore a mapping function for a simply-connected region $R$ of the $\zeta$-plane. Now in the theory of conformal mapping, simply-connected regions are often classified according to how much is assumed about the nature of the boundary, the classification running as follows: general simplyconnected regions (prime end theory), simply-connected regions all of whose boundary points are attainable, and Jordan regions $\left({ }^{3}\right)$. The corresponding mapping functions are respectively univalent in $|z|<1$, continuous on $|z| \leqq 1$ and univalent in $|z|<1$, and continuous and univalent in $|z| \leqq 1$. We shall

(3) A Jordan region is a region bounded by a Jordan curve. A Jordan curve is a simple closed curve; i.e., a one-to-one continuous map of a circle. A Jordan arc is a continuous one-to-one map of the closed unit interval $[0,1]$. 
deduce a different form for the decomposition theorem corresponding to each of these classes of regions (or classes of mapping functions); and also a form corresponding to the class of Jordan regions bounded by analytic curves (the class of mapping functions $\zeta=f_{1}(z)$ regular and univalent on $|z| \leqq 1$ ). Two of these theorems are direct corollaries of the theorem for the most general case, and will be treated with that theorem in $\$ 3$. But the theorem corresponding to Jordan regions is deeper and requires considerable development.

This development is suggested by the rather remarkable theorem (which together with related theorems, we shall discuss in $\$ 4$ ) that if $f(z)$ is regular in $|z|<1$, and if the limiting values of $f(z)$ as $|z| \rightarrow 1$ all lie on a Jordan curve $\Gamma$ of the $w$-plane, then $f(z)$ can be decomposed as $f(z) \equiv f_{2}\left(f_{1}(z)\right)$, where $\zeta=f_{1}(z)$ is a rational function of the form

$$
f_{1}(z) \equiv \prod_{j=1}^{n} \frac{z-a_{j}}{1-\bar{a}_{j} z}, \quad\left|a_{j}\right|<1
$$

and $f_{2}(\zeta)$ is a mapping function for the inner Jordan region bounded by $\Gamma$. The rational functions of the form

$$
w=f(z) \equiv K \prod_{j=1}^{n} \frac{z-a_{j}}{1-\bar{a}_{j} z}, \quad\left|a_{j}\right|<1,
$$

will occur of ten in this paper, and we shall call them circular rational functions. They are the functions $\left(^{4}\right)$ which map the unit circle $|z| \leqq 1$ onto the circle $|w| \leqq|K|$ with each value taken precisely $n$ times.

One result of the above theorem is that $f(z)$ is continuous on the closed circle $|z| \leqq 1$, and a second result is that $f(z)$ is power-multivalent (in fact, locally univalent) along $|z|=1$. These conclusions suggest the following possible generalizations. First, if $f(z)$ is meromorphic in $|z|<1$, and if the limiting values of $f(z)$ as $z$ approaches the points of an $\operatorname{arc} \gamma$ of $|z|=1$ lie on a Jordan arc $\Gamma$ of the $w$-plane, then $f(z)$ is continuous on $\gamma$. Second, under the same circumstances, if $f(z)$ is continuous on $\gamma$ then $f(z)$ is power-multivalent along $\gamma$. In $\$ 5$ we shall prove both of these statements, and from them, together with an earlier theorem, we shall have at once our best decomposition theorem (corresponding to the class of Jordan regions).

We have not yet considered the rational function $f_{2}(\zeta)$ in any detail. It is fairly evident that in the cases considered so far (i.e., those involving $f(z)$ meromorphic on $|z|<1$ ), nothing can be inferred about the nature of $f_{2}(\zeta)$ beyond the fact that it is rational. But if $f(z)$ is regular and bounded on $|z|<1$, then certain of the decomposition theorems can be proved for restricted classes of rational functions. To be explicit, all of the theorems whose premises include (perhaps implicitly) the continuity of $f(z)$ on $|z| \leqq 1$ can be

(4) See, for example, Julia, Principes Géométriques d'Analyse, vol. 1, p. 54. 
proved with $f_{2}(\zeta)$ restricted either to polynomials or to circular rational functions. The general theorem, however, cannot be proved in this case, as we shall show by an example. These theorems and this example will be taken up in $\$ 6$.

Before proceeding to the first theorem let us recall a few function-theoretic facts.

First, the surfaces which we have called rational surfaces are precisely the surfaces composed of $n$ extended planes joined around branch points whose total order is $2 n-2$. For every rational surface has this construction $\left(^{\mathfrak{b}}\right)$. And conversely, any such surface is simply-connected (having an EulerPoincaré characteristic of -2 as any subdivision will show) $\left.{ }^{6}\right)$, and hence can be mapped by the fundamental mapping theorem onto a normal region $\left({ }^{7}\right)$, which must.in this case be the extended plane. But any function meromorphic on the extended plane is rational.

Second, the Riemann configurations onto which the circular rational functions map the unit circle $|z| \leqq 1$ (we shall call them surfaces of type II) are precisely those configurations composed of $n$ circular discs $|w| \leqq M$ joined around branch points whose total order is $n-1$. For every surface of type II has this construction $\left({ }^{8}\right)$. And conversely, any such surface is simply-connected (with characteristic -1$)\left({ }^{9}\right)$ and hence mappable onto a normal region which in this case must be a circle. But any function which maps the unit circle onto $|w|<M$ with each value taken precisely $n$ times is a circular rational function $\left({ }^{10}\right)$.

We shall also assume one or two results centering around the theorem of Fatou $\left.{ }^{11}\right)$ that if $f(z)$ is regular and bounded on $|z|<1$, then the radial limit $\lim _{r \rightarrow 1} f\left(r e^{i \theta}\right)$ exists for almost all $\theta$. A direct corollary affirms the same result for $f(z)$ univalent on $|z|<1$. Also in this connection we note the theorem of F. and M. Riesz $\left({ }^{12}\right)$ that if $f(z)$ is regular and bounded and not identically constant, then the points of $|z|=1$ where the radial limit is zero form a set of Lebesgue measure zero. Finally, by a theorem of Lindelöf $\left({ }^{13}\right)$, if $f(z)$ is

(5) See, for example, Julia, loc. cit., p. 60.

${ }^{(6)}$ See Kerékjárto, Vorlesungen über Topologie, I, pp. 143-147.

(7) The difficult fundamental theorem of uniformization is not actually needed here. It is sufficient to divide the surface in question into a finite number of plane pieces, and apply the theorem on the blending of the maps of contiguous or overlapping regions. See, for example, Bieberbach, Funktionentheorie, vol. 2, p. 171.

(8) See Julia, loc. cit., pp. 54-56, 60. The rational surface of a circular rational function is symmetric with respect to inversion in the circle $|w|=|K|$, and any rational surface of $n$ sheets has branch points of total order $2 n-2$.

(9) See Footnote 6.

(10) See Footnote 4.

(11) See Bieberbach, loc. cit., pp. 147-155.

(12) See Bieberbach, loc. cit., pp. 155-156.

(13) See Bieberbach, loc. cit., p. 21. 
regular and bounded on $|z|<1$ and if $\lim _{z \rightarrow z_{0}} f(z)$ exists along two Jordan arcs lying in $|z|<1$ except for the common endpoint $z_{0}$, then the two limits are the same and $f(z)$ uniformly approaches the limit between the two arcs.

A Jordan arc which lies in a region $R$ except for a single endpoint which lies on the boundary of $R$ is known as a Jordan cut of $R$. A Jordan arc which lies in $R$ except for both endpoints which lie on the boundary of $R$ is known as a Jordan crosscut of $R$. On a general Riemann configuration $W$ a Jordan cut will be defined as a semi-open Jordan arc, whose open end converges to the boundary of $W$ in such a way that its projection on the extended $w$-plane converges to a point.

We need to make one more general remark. As long as we consider meromorphic functions, such notions as limit, continuity, Jordan arc, etc., must be extended to allow infinite values. This extension is the usual one, geometrized by projecting to the Riemann sphere, and considering limits, continuity, Jordan arcs, and so on, in the ordinary sense for the corresponding sets of points there.

3. The fundamental decomposition theorem. We prove first a lemma.

Lemma 1. If a Riemann configuration $W$ can be divided by a Jordan crosscut into two Riemann configurations each of which can be imbedded in the Riemann surface of the inverse of a rational function, then $W$ itself can be so imbedded.

Proof. Let $\gamma$, with endpoints $A$ and $B$, be the crosscut dividing $W$ in to $W_{1}$ and $W_{2}$, and let $W_{1}$ and $W_{2}$ be imbedded in rational surfaces $R_{1}$ and $R_{2}$ respectively. Cut each of $R_{1}$ and $R_{2}$ along $\gamma$, and join the left of $\gamma$ on $R_{1}$ to the right of $\gamma$ on $R_{2}$, and vice versa. (Here left and right are used simply to designate the two sides of $\gamma$.)

It is obvious that the above construction can change branch point order only on $\gamma$. Let $p$ and $q$ be the orders of the branch points at $A$ of $R_{1}$ and $R_{2}$ respectively, with the convention that, for instance, $p=0$ if $R_{1}$ has no branch point at $A$. Then $R_{1}$ and $R_{2}$ have respectively $p+1$ and $q+1$ sheets joined around $A$. The new surface $R$ has $p+q+2$ sheets joined around $A$, i.e., has a branch of order $p+q+1$ at $A$. Now consider an interior point $C$ of $\gamma$. The construction given carries the two copies $\gamma_{1}$ and $\gamma_{2}$ of $\gamma$ on $R_{1}$ and $R_{2}$ into two copies forming a closed curve on $R$. The two points $C_{1}$ and $C_{2}$ on $\gamma_{1}$ and $\gamma_{2}$ correspond to distinct points of $R$. Hence the total order of the two branch points remains the same. For, although the branch points at both $C_{1}$ and $C_{2}$ may be changed in the construction, the total number of sheets joined around these two points does not change. Thus the method of joining $R_{1}$ and $R_{2}$ defined above has increased by 2 the total order of the branch points over that for the two surfaces separately (an increase of 1 at each of $A$ and $B$ ).

Let the number of sheets of $R_{1}$ and $R_{2}$ be $m$ and $n$ respectively. Then, since $R_{1}$ and $R_{2}$ are rational, they have branch points of total order $2 m-2$ and $2 n-2$ respectively. Hence on the new surface $R$ the total order of the 
branch points is $2(m+n)-2$. Thus $R$ is rational and the lemma is proved.

In the next proof we shall use the fact that $R$ has points exterior to $W$. For instance, one of the two copies of $\gamma$ on $R$ crosscuts $W$ and the other lies outside of $W$.

THEOREM 1. If $f(z)$ is meromorphic in $|z|<1$, then a necessary and suffcient condition that it can be decomposed, $f(z) \equiv f_{2}\left(f_{1}(z)\right)$, where $f_{2}(\zeta)$ is a rational function and $\zeta=f_{1}(z)$ is regular, bounded and univalent on $|z|<1$ is that $f(z)$ be rationally-multivalent at every point of $|z|=1$.

Proof. We need consider only the sufficiency. If $f(z)$ is rationally multivalent along $|z|=1$ then every point of $|z| \leqq 1$ has a neighborhood (halfneighborhood for points of $|z|=1$ ) whose map under $w=f(z)$ can be imbedded on a rational surface. By the Heine-Borel theorem we can choose a finite number of these neighborhoods and half-neighborhoods covering $|z|<1$. If $g_{2}(\zeta)$ is a rational function such that $\zeta=g_{2}^{-1}(f(z))$ is univalent in one of these neighborhoods, then for almost all boundary points of this neighborhood lim $g_{2}^{-1}(f(z))$ exists as $z$ approaches the boundary along the interior normal. Thus the radial $\operatorname{limit}_{\lim } \rightarrow f\left(r e^{i \theta}\right)$ exists for almost all $\theta$. We can therefore effect a decomposition of $|z|<1$ into a finite number of cells, bounded by Jordan arcs and Jordan cuts of $|z|<1$, such that the maps of the latter under $w=f(z)$ are Jordan arcs and Jordan cuts of the Riemann configuration $W$ onto which $w=f(z)$ maps the circle $|z|<1$. These divide $W$ into a finite number of simply-connected Riemann configurations (the maps of the cells of $|z|<1$ ) each of which can be imbedded on a rational surface. By an induction on Lemma $1, W$ itself can be imbedded on a rational surface $R$. Let $f_{2}(\zeta)$ be a rational function mapping the extended $\zeta$-plane onto $R$. Then $f_{1}(z) \equiv f_{2}^{-1}(f(z))$ is univalent on $|z|<1$, and $f(z) \equiv f_{2}\left(f_{1}(z)\right)$. Since $R$ contains points exterior to $W$, the function $f_{2}(\zeta)$ can be chosen so that $f_{1}(z)$ is regular and bounded in $|z|<1$.

In each of the following two theorems one part is trivial, while the other part is an immediate consequence of Theorem 1.

THEOREM 2. If $f(z)$ is meromorphic in $|z|<1$, and continuous in $|z| \leqq 1$, then a necessary and sufficient condition that it can be decomposed as in Theorem 1 is that $f(z)$ be power-multivalent at every point of $|z|=1$.

TheOREM 3. A necessary and sufficient condition that $f(z)$ be meromorphic in $|z| \leqq 1$ is that $f(z)$ be decomposable as in Theorem 1 with $f_{1}(z)$ regular and univalent in $|z| \leqq 1$.

Theorem 2 follows from the fact that if $f(z)$ is continuous on $|z| \leqq 1$ then rational-multivalency along $|z|=1$ implies power-multivalency. For if the map of a half-neighborhood of $z_{0}\left(\left|z_{0}\right|=1\right)$ can be imbedded in a rational surface $R$, then, by the continuity of $f(z)$ at $z_{0}$, a small enough half-neighborhood will involve at most one branch point of $R$. Theorem 3 is even more direct, 
for a function is power-multivalent at any point where it is meromorphic, and thus $f(z)$ is power-multivalent on the closed circle $|z| \leqq 1$.

If we view the decomposition of $f(z)$ with regard to the kind of plane simply-connected region for which $\zeta=f_{1}(z)$ is a mapping function, then Theorems 1,2 and 3 correspond in that order to general simply-connected regions, simply-connected regions all of whose boundary points are accessible (attainable), and Jordan regions bounded by analytic curves. The corresponding theorem for general Jordan regions will be developed in $\$ 5$.

THEOREM 4. If $f(z)$ is meromorphic in $|z|<1$, then a necessary and suffcient condition that $f(z)$ can be decomposed as in Theorem 1 is that there exist a univalent function $z=f_{3}(\zeta)$ mapping a simply-connected region $G$ of the $\zeta$-plane onto $|z|<1$ such that $f\left(f_{3}(\zeta)\right)$ is meromorphic in a simply-connected region $G_{1}$ of the $\zeta$-plane containing the closure $\bar{G}$ of $G$.

Proof. The condition is obviously necessary; we need only take $f_{3}(\zeta) \equiv f_{1}^{-1}(\zeta)$. On the other hand, suppose that there exists such a function $z=f_{3}(\zeta)$. Consider an analytic Jordan curve $J$ in $G_{1}$ containing $\bar{G}$ in its interior. (We can find $J$ by mapping $G_{1}$ onto the unit circle $|z|<1$. The map of $\bar{G}$ is bounded away from the circumference and hence is interior to some circle $|z|=r<1$; then $J$ may be taken as the image of $|z|=r$.) We have $f\left(f_{3}(\zeta)\right)$ meromorphic on and within $J$, and thus by Theorem 3 we can find a decomposition $f\left(f_{3}(\zeta)\right) \equiv f_{2}\left(g_{1}(\zeta)\right)$ where $f_{2}$ and $g_{1}$ are rational and univalent respectively. Then

$$
f(z) \equiv f_{2}\left(g_{1}\left(f_{3}^{-1}(z)\right)\right) \equiv f_{2}\left(f_{1}(z)\right)
$$

where $f_{1}(z) \equiv g_{1}\left(f_{3}^{-1}(z)\right)$ is univalent on $|z|<1$.

4. Miscellaneous decomposition theorems. In this section we shall prove two interesting theorems which are, in a certain sense, natural predecessors of the theorems of the next section.

THEOREM 5. If $f(z)$ is meromorphic in $|z|<1$, and if the set $S$ of limiting values of $f(z)$ as $|z| \rightarrow 1$ contains no value assumed by $f(z)$ in $|z|<1$, then $f(z)$ can be written $f(z) \equiv f_{2}\left(f_{1}(z)\right)$ where $\zeta=f_{1}(z)$ is a circular rational function, and $w=f_{2}(z)$ is a mapping function for a simply-connected region in the w-plane whose boundary is precisely the set $S$.

Proof. Let $O$ be the set of values assumed by $f(z)$ in $|z|<1$. By hypothesis the boundary of $O$ is $S$; moreover $S$ is a continuum. Therefore $O$ is a simplyconnected region of the $w$-plane. If $\zeta=f_{2}^{-1}(w)$ maps $O$ conformally onto $|\zeta|<1$, then $\zeta=f_{1}(z) \equiv f_{2}^{-1}(f(z))$ maps $|z|<1$ into $|\zeta|<1$ and the set of limiting values of $\zeta=f_{1}(z)$ as $|z| \rightarrow 1$ lies on $|\zeta|=1$. From this it follows that $f_{1}(z)$ is a circular rational function. For $f_{1}(z)$ can have only a finite number of zeros in $|z|<1$, and if $g(z)$ is a circular rational function having the same set of zeros, then $\zeta=f_{1}(z) / g(z)$ is a regular function which does not vanish in $|z|<1$, and whose limiting values as $|z| \rightarrow 1$ again lie on $|\zeta|=1$. But this function 
must be identically constant. Thus $\zeta=f_{1}(z)$ is a circular rational function, and $f(z) \equiv f_{2}\left(f_{1}(z)\right)$, which completes the proof of the theorem.

Now if $f(z)$ is regular in $|z|<1$, and if its limiting values lie on a Jordan curve of the $w$-plane, we do not need to assume that the limiting set contains no value taken on by $f(z)$ in $|z|<1$. The proof of this theorem depends on the following geometric formulation of the principle of maximum modulus:

If $f(z)$ is regular in $|z|<1$, and if the limiting values of $f(z)$ as $|z| \rightarrow 1$ form a bounded set $S$ in the w-plane, then every value which $f(z)$ assumes in $|z|<1$ is separated by $S$ from infinity.

For if a value $w_{0}=f\left(z_{0}\right)$ with $\left|z_{0}\right|<1$ is not separated from infinity by $S$, there is an arc joining $w_{0}$ to $\infty$ which contains no point of $S$ save perhaps the endpoint $w_{0}$. If $w$ traces this arc starting from $w_{0}$, then $z$ traces an arc lying in $|z|<1$, which contradicts the boundedness of $f(z)$.

THeorem 6. If $f(z)$ is regular in $|z|<1$, and if the set $S$ of limiting values of $f(z)$ as $|z| \rightarrow 1$ lies on a Jordan curve $\Gamma$ of the w-plane, then $f(z)$ can be expressed $f(z) \equiv f_{2}\left(f_{1}(z)\right)$ where $\zeta=f_{1}(z)$ is a circular rational function, and $w=f_{2}(\zeta)$ is a mapping function for the interior of $\Gamma$.

For all the points of $\Gamma$ are accessible from infinity, and thus, by the geometric principle formulated above, the values of $f(z)$ in $|z|<1$ lie inside $\Gamma$. If $\zeta=f_{2}^{-1}(w)$ maps this interior Jordan region onto $|\zeta|<1$, we have just as in Theorem 5 that $\zeta=f_{1}(z) \equiv f_{2}^{-1}(f(z))$ is a circular rational function, and that $f(z) \equiv f_{2}\left(f_{1}(z)\right)$.

CoROllary 1. In either of the above two theorems if some value is assumed by $f(z)$ precisely once in $|z|<1$, or is a limiting value of $f(z)$ at precisely one point of $|z|=1$, then $w=f(z)$ is a mapping function for a simply-connected region of the w-plane whose boundary is the set $S$.

CoROllaRy 2. In either of the above two theorems $f(z)$ can be expressed as in Theorem 1 as a rational function of a mapping function.

Corollary 1 is obvious, and Corollary 2 follows from the fact that $f(z)$ is locally univalent along $|z|=1$ in both theorems.

5. Decompositions involving mapping functions for Jordan regions. In this section we shall consider the generalization of Theorem 6 in which the conditions of that theorem are assumed on only part of the circumference $|z|=1$.

THEOREM 7. If $f(z)$ is meromorphic in $|z|<1$, and if the limiting values of $f(z)$ as $|z| \rightarrow 1$ along an arc $\gamma$ of $|z|=1$ lie on a Jordan arc $\Gamma$ of the w-plane, then $f(z)$ is continuous on the interior of $\gamma$.

Proof. Let $z_{0}$ be an interior point of $\gamma$ and let $w_{0}$ be a limiting value of $f(z)$ 
at $z_{0}$. Choose a sequence $\left\{z_{i}\right\}$ in $|z|<1$, converging to $z_{0}$, and having the following properties: (1) $f\left(z_{i}\right) \rightarrow w_{0},(2)$ all $f\left(z_{i}\right)$ lie off $\Gamma$ and on the same side of $\Gamma$ (if $w_{0}$ is an endpoint of $\Gamma$ this condition is vacuous), (3) no $f\left(z_{i}\right)$ is a value of $f(z)$ at a zero of $f^{\prime}(z)$. All of this can be gotten from an original sequence satisfying (1) by slight displacements and the selection of a subsequence.

Let $J$ be a Jordan arc of the w-plane having no point in common with $\Gamma$ except for an endpoint at $w_{0}$, and let $J$ contain all the $f\left(z_{i}\right)$ and contain no value $w^{\prime}$ assumed by $f(z)$ at a zero of $f^{\prime}(z)$. (This last is possible because such values $w^{\prime}$ are at most denumerable and can accumulate only on $\Gamma$.)

Now consider a half-neighborhood $R$ of $z_{0}$, and its bounding arc $\gamma^{\prime}$ on $\left|z-z_{0}\right|=r$. By Fatou's theorem and the theorem of F. and M. Riesz, we may take $r$ so that at the endpoints of $\gamma^{\prime}, f(z)$ has definite radial limits, different from each other and from $w_{0}$. But then there are at most a finite number of points on $\gamma^{\prime}$ where $f(z)$ takes the value $w_{0}$, and we remove these by changing $\gamma^{\prime}$ to a new circular arc with the same endpoints, lying in $R$ sufficiently close to $\gamma^{\prime}$. We shall call this new arc and its neighborhood of $z_{0}$ respectively $\gamma^{\prime}$ and $R$.

Since $f\left(\gamma^{\prime}\right)$ does not contain $w_{0}$, it has no point in common with a sufficiently small circle $\left|w-w_{0}\right|<\rho$. All of $J$ from some point on lies in this circle, and contains the maps $f\left(z_{i}\right)$ of all $z_{i}$ from some $i$ on. Let $J$ now denote this subarc, and the $z_{i}$ this subsequence. Then no point of $J$ except $w_{0}$ is a limiting value of $f(z)$ as $z$ approaches the boundary of $R$. Therefore, starting at each $z_{i}$, as $f(z)$ traces $J$ in both directions from $f\left(z_{i}\right)$ the point $z$ traces in $R$ a simple arc $J_{i}$ containing $z_{i}$. Since all the limiting values of $f(z)$ lie on $\Gamma$, there can be at most a finite number of the $J_{i}$, and some one, say $J_{1}$, contains an infinite number of the $z_{i}$. But then $J_{1}$ is a Jordan arc with endpoint $z_{0}$, because, by just the argument we have been giving, every half-neighborhood of $z_{0}$ contains all of $J_{1}$ from some point on.

Thus for every limiting value $w_{0}$ at $z_{0}$ there is a Jordan cut $J$ to $z_{0}$ such that as $z \rightarrow z_{0}$ on $J, f(z) \rightarrow w_{0}$. But then all limiting values at $z_{0}$ are the same (Lindelöf) and $f(z)$ is continuous at $z_{0}$, which proves the theorem.

The following fact will prove useful in the next theorem. If $w_{0}$ is not an endpoint of $\Gamma$, let $J^{\prime}$ be a Jordan curve lying in the circle $\left|w-w_{0}\right|<\rho$, such that a subarc of $\Gamma$ containing $w_{0}$ crosscuts the interior of $J^{\prime}$ and divides it into two Jordan regions $R_{1}$ and $R_{2}$. We assert that the map of $R$ under $f(z)$ covers at least one of $R_{1}$ and $R_{2}$. For no limiting value of $f(z)$ along the boundary of $R$ lies in $R_{1}$ or $R_{2}$. And $R$ contains points (of $J_{1}$ ) mapping into one of $R_{1}$ and $R_{2}$.

THEOREM 8. If $f(z)$ is meromorphic in the unit circle $|z|<1$ and continuous on a closed arc $\gamma$ of the circumference $|z|=1$, and if the values which $f(z)$ assumes on $\gamma$ lie on a Jordan arc $\Gamma$ of the w-plane, then $f(z)$ is power-multivalent at every point of $\gamma_{1}$, the interior arc of $\gamma$. 
Proof. Our general scheme of proof here is to continue $f(z)$ over $\gamma_{1}$ in such a way that the new function $F(z)$, defined on a region containing $\gamma_{1}$, is an interior transformation. The power-multivalency of $f(z)$ along $\gamma_{1}$ is an immediate consequence of this property.

Let $R_{w}$ be a Jordan region in the $w$-plane which contains $\Gamma$ as a crosscut. Let $\gamma^{\prime}$ be a Jordan crosscut of $|z|<1$ having the same endpoints as $\gamma$, and let $\gamma^{\prime}$ be chosen so that, in the Jordan region $R_{z}^{\prime}$ bounded by $\gamma$ and $\gamma^{\prime}, f(z)$ takes only values lying in $R_{w}$. (We can do this as follows. Since $\Gamma$ can always be made a little longer, we can assume that only interior points of $\Gamma$ are covered by $f(z)$ on $\gamma_{1}$. But then, by the continuity of $f(z)$ on $\gamma$, each point of $\gamma_{1}$ has a half-neighborhood whose map lies in $R_{w}$; and we can draw $\gamma^{\prime}$ on these half-neighborhoods.) Let $R_{z}^{\prime \prime}$ be the reflection of $R_{z}^{\prime}$ in $|z|=1$, and let $R_{z}$ be the Jordan region $R_{z}^{\prime}+\gamma_{1}+R_{z}^{\prime \prime}$. We now define a function $F(z)$ over $R_{z}$ as follows. Let the two Jordan regions into which $\Gamma$ divides $R_{w}$ be $R_{w}^{\prime}$ and $R_{w}^{\prime \prime}$, and consider a homeomorphism between the closures of $R_{w}{ }^{\prime}$ and $R_{w}{ }^{\prime \prime}$ which leaves $\Gamma$ invariant. Let the value of $F(z)$ at a point of $R_{z}^{\prime \prime}$ be defined as the homeomorph of the value of $f(z)$ at the inverse point in $R_{z}^{\prime}$. And if $z$ lies in $R_{z}^{\prime}+\gamma_{1}$, let $F(z)=f(z)$.

We shall show that $w=f(z)$ is an interior transformation. First, $F(z)$ is obviously continuous. Second, $F(z)$ maps no continuum of $R_{z}$ onto a point $w_{1}$ of $R_{w}$. For the set of points of $R_{z}^{\prime}+R_{z}^{\prime \prime}$ which map onto $w_{1}$ is isolated in $R_{z}^{\prime}+R_{z}^{\prime \prime}$ (by the definition of $F(z)$ and the analyticity of $f(z)$ in $R_{z}^{\prime}$ ). And if $f(z)$ is not identically constant, the set of points on $\gamma_{1}$ which map onto $w_{1}$ is nondense on $\gamma_{1}$. Finally, $F(z)$ takes any open set in $R_{z}$ into an open set of $R_{w}$. For the map of a neighborhood of $z$ (in $R_{z}$ ) contains a neighborhood (in $R_{w}$ ) of $F(z)$. This follows for $z$ in $R_{z}^{\prime}+R_{z}^{\prime \prime}$ from the analyticity of $f(z)$ in $R_{z}^{\prime}$ and the definition of $F(z)$, and for $z$ on $\gamma_{1}$ from the remark at the end of the previous theorem and the definition of $F(z)$. But then $F(z)$ has the properties which define an interior transformation; and therefore, by the fundamental theorem on interior transformations $\left.{ }^{14}\right) F(z)$ has at every point of $R_{z}$ the property which we have called power-multivalency. In particular, $f(z)$ is power-multivalent along $\gamma_{1}$ (with respect to half-neighborhoods in $|z|<1$ ).

We have at once from Theorems 1, 7 and 8:

THEOREM 9. If $f(z)$ is meromorphic in $|z|<1$, then a necessary and sufficient condition that $f(z)$ be decomposable as in Theorem 1 , where $\zeta=f_{1}(z)$ is a mapping function for a Jordan region of the $\zeta$-plane, is that for every $z_{0}$ on $|z|=1$ there is an integer $n$ and an arc $\gamma$ of $|z|=1$ containing $z_{0}$, such that for some $w_{0}$ the limiting values of $\left[f(z)-w_{0}\right]^{1 / n}$ as $|z| \rightarrow 1$ along $\gamma$ lie on a Jordan arc $\Gamma$ of the $w$-plane ( $w_{0}$ and $\Gamma$ depending on $z_{0}$ ).

It will be easily verified that Theorems 7 and 8 remain valid if we replace

(14) See Stoïlow, Principes Topologiques de la Théorie des Fonctions Analytiques, pp. 108-116. 
the single arc $\Gamma$ by a finite collection of Jordan arcs having only a finite number of points of intersection. Theorem 9 could of course be rephrased in this respect, but Theorem 9 is complicated enough already, and we shall use this generalization only in the following theorem.

THEOREM 10. If $f(z)$ is meromorphic on $|z|<1$, and if the limiting values of $f(z)$ as $|z| \rightarrow 1$ all lie on a closed curve of the w-plane with at most a finite number of self-intersections, then $f(z)$ can be decomposed as in Theorem 1 , where $\zeta=f_{1}(z)$ is a mapping function for a Jordan region.

This theorem follows immediately from Theorem 1 and from Theorems 7 and 8 in the more general form mentioned above.

We close this section with the generalization of Theorem 5 in which the conditions of that theorem are assumed only locally along the circumference $|z|=1$. With these weakened assumptions we can still effect, as in Corollary 2 of that theorem, a decomposition of the kind in Theorem 1.

THEOREM 11. If $f(z)$ is meromorphic in $|z|<1$, and if for every $z_{0}$ on $|z|=1$ there is a half-neighborhood $R$ with boundary arc $\gamma$ on $|z|=1$, such that the set of limiting values of $f(z)$ as $z \rightarrow \gamma$ in $R$ contains no value assumed by $f(z)$ in $R$, then $f(z)$ can be decomposed as $f(z) \equiv f_{2}\left(f_{1}(z)\right)$ where $\zeta=f_{1}(z)$ is a univalent function mapping $|z|<1$ conformally onto a bounded simply-connected region $G$ of the $\zeta$-plane, and $f_{2}(\zeta)$ is a rational function which is locally univalent (no multiple pole of $f_{2}(\zeta)$ or zero of $f_{2}^{\prime}(\zeta)$ ) on the boundary of $G$.

Proof. The half-neighborhood $R$ is bounded by the arc $\gamma$ of $|z|=1$ and a circular arc $\gamma^{\prime}$ of $\left|z-z_{0}\right|=r$. By hypothesis we may assume that the values of $f(z)$ on the interior of $\gamma^{\prime}$ are distinct from the limiting values of $f(z)$ along $\gamma$. The latter set forms a continuum $S$ of the $w$-plane which contains no point of the set $O$ mapping from $R$. Then $O$ lies in a simply-connected region $O^{\prime}$ bounded by $S$, and if $w=f_{2}(\zeta)$ maps the unit circle $|\zeta|<1$ onto $O^{\prime}$, then the limiting values of $\zeta=f_{1}(z) \equiv f_{2}^{-1}(f(z))$ along $\gamma$ lie on the Jordan curve $|\zeta|=1$. By Theorems 7 and $8, f_{1}(z)$ is continuous and power-multivalent along $\gamma$, and since by hypothesis $f(z)$ assumes no value in $R$ which it assumes on $\gamma, f_{1}(z)$ is locally univalent along the interior of $\gamma$. Hence $f(z)$ is locally univalent along $\gamma$, and likewise along the whole of $|z|=1$. But then the required decomposition is possible by Theorem 1 .

6. The function $f(z)$ regular and bounded in $|z|<1$. In this section we shall consider the decomposition of $f(z)$ with respect to special classes of rational functions. It is evident that we can make no restriction on $f_{2}(\zeta)$ so long as we leave $f(z)$ meromorphic on $|z|<1$. But if we consider $f(z)$ regular and bounded in $|z|<1$, then in certain cases we can confine $f_{2}(\zeta)$ to polynomials or to circular rational functions, as we shall proceed to show.

LemMA 2. If a simply-connected Riemann configuration $W$ can be divided by a crosscut into two Riemann configurations each of which can be imbedded in 
a surface of type II, and if all the boundary points of $W$ are attainable, then $W$ itself can be so imbedded.

Proof. This lemma is similar to Lemma 1 but more difficult. Let $\gamma$ with endpoints $A$ and $B$ be the crosscut dividing $W$ into $W_{1}$ and $W_{2}$, and let $W_{1}$ and $W_{2}$ be imbedded in surfaces (of type II) $R_{1}$ and $R_{2}$ respectively. We shall take $R_{1}$ and $R_{2}$ over the same circle $|w| \leqq M$ of the $w$-plane. If we join $R_{1}$ and $R_{2}$ just as in Lemma 1 , we shall have $W$ imbedded in a disc surface $R$ which has one too many branch points to be a surface of type II.

Let $J_{1}$ and $J_{2}$ be Jordan cuts on $R_{1}$ and $R_{2}$ respectively which run from $B$ to the circumference $|w|=M$ and have no points in common with $W_{1}$ and $W_{2}$ respectively. Such $J_{1}$ and $J_{2}$ exist, for if a bounded plane simply-connected region $G$ has all its boundary points attainable, then every boundary point of $G$ can be joined to infinity along a Jordan arc having no point in common with $G$. In $R, J_{1}+J_{2}$ forms a non-dividing Jordan crosscut. If we can find a surface $R^{\prime}$ of type II which also contains $J_{1}+J_{2}$ as a Jordan crosscut, then by cutting each surface along $J_{1}+J_{2}$ and joining the left of $J_{1}+J_{2}$ on $R$ to its right on $R^{\prime}$ and vice versa, we construct a new surface which contains $W$ and which is obviously of type II.

To find such a surface $R$ we consider again $R_{1}$ and $R_{2}$. The surface $R_{1}$ contains $J_{1}$ and $R_{2}$ contains $J_{2}$. Now if we can find a cut $J$ which lies on each of $R_{1}$ and $R_{2}$ and runs from $B$ to the boundary, and which touches $J_{1}$ and $J_{2}$ only at $B$, then by cutting $R_{1}$ and $R_{2}$ along $J$ and joining them together around $B$ (the left of $J$ on $R_{1}$ to its right on $R_{2}$ and vice versa) the resulting surface will be of type II and will contain $J_{1}+J_{2}$ as a Jordan crosscut. We accordingly seek such a $J$.

Let $C$ be a point on $\gamma$ having maximum distance from $B$ (measured on the $w$-plane) and let $J^{\prime \prime}$ be the straight line segment from $C$ to the boundary $|w|=M$ in the direction of a line from $B$ to $C$. (If $J^{\prime \prime}$ meets a branch point of $R_{1}$ it may be continued on any one sheet joined there.) Now if $J^{\prime}$ is the arc of $\gamma$ between $B$ and $C$, then the Jordan cut $J=J^{\prime}+J^{\prime \prime}$ lies on both $R_{1}$ and $R_{2}$. But in general $J=J^{\prime}+J^{\prime \prime}$ will intersect $J_{1}$ on $R_{1}$ at points other than $B$.

We avoid this difficulty by suitably enlarging $R_{1}$. If we cut $R_{1}$ along $J$ and join it to a model of $R_{1}$ similarly cut (the left of $J$ on $R_{1}$ to its right on the model, and vice versa) we get a surface of type II containing two images each of $J_{1}$ and $J$. We assert that if $n$ models of $R_{1}$ be added to $R_{1}$ in this way, each cut along $J$, and all joined cyclically around $B$ (the left of $J$ on $R_{1}$ to its right on the first model, its left on the first model to its right on the second model, and so on until finally its left on the $n$th model to its right on $R_{1}$ ) then for some in teger $n$ the resulting surface of type II, $R_{1}^{\prime}$, contains a pair $J_{1}$ and $J$ having only the point $B$ in common.

For on $R_{1}, J_{1}$ does not cross $J^{\prime}$ (since the points on one side of $J^{\prime}$ lie in $W_{1}$, while $J_{1}$ is outside of $W_{1}$ ). Hence a subarc of $J_{1}$ between two intersections of $J_{1}$ 
with $J^{\prime \prime}$ has a definite integral order on $R_{1}$ with respect to $J^{\prime}$, and since $J_{1}$ is a Jordan arc, there is an integer $N$ which is greater than the order of every such subarc. If $N$ models of $R_{1}$ are added as described above, the resulting surface (of type II) has $N+1$ copies of $J$ which are ordered cyclically so that if a given copy of $J_{1}$ meets the $n$th and the $(n+j)$ th copies of $J^{\prime \prime}$, then on $R_{1}$ a subarc of $J_{1}$ has order of at least $j$ with respect to $J^{\prime}$. But then there is some copy of $J^{\prime \prime}$, and hence of $J=J^{\prime}+J^{\prime \prime}$, which the given copy of $J_{1}$ does not meet.

Similarly we find a surface of type II, $R_{2}^{\prime}$, containing $J=J^{\prime}+J^{\prime \prime}$ and $J_{2}$ with only the point $B$ in common. If we now join $R_{1}^{\prime}$ and $R_{2}^{\prime}$ as indicated earlier, we have the required surface $R^{\prime}$, which completes the proof of the lemma.

THEOREM 12. If $f(z)$ is regular and bounded in $|z|<1$ then Theorem 2 holds with $f_{2}(\zeta)$ taken either as a polynomial or as a circular rational function.

The proof for the representation of $f(z)$ in terms of the circular rational function follows from Lemma 2 just as the proof of Theorem 1 followed from Lemma 1 , and consists mainly in showing that the Riemann configuration $W$ onto which $w=f(z)$ maps the unit circle $|z|<1$ can be imbedded in a surface of type II. But any surface of type II can be completed to a rational surface of a polynomial by adding the exteriors of the $n$ discs $|w| \leqq M$ joined around a single branch point of order $n-1$ at infinity. Therefore the Riemann configuration $W$ can be imbedded in a rational surface of a polynomial, and this completes the proof of the theorem.

Classified by the type of mapping function occurring in the decomposition, this theorem corresponds to Theorem 2 rather than to Theorem 1 . The analogues of Theorems 3 and 10 are also theorems, and follow immediately from Theorem 12 (and Theorems 8 and 9). The analogue of Theorem 1, however (and hence also the analogue of Theorem 4), is not a theorem, as we shall show by the following example.

Consider the circles $|w \pm i|=1$ on the $w$-plane. If both are cut at $w=0$, and are suitably placed on the rational surface $R$ having two sheets and branch points at $\pm i$, they can be joined to form a simple smooth closed curve which is described like a figure-eight. Now consider the circles $|w \pm i|=1 \pm \frac{1}{2}$. On $R$ these can be taken to form a second figure-eight, having no point in common with the first, such that the two together bound a band $S$ in the shape of a figure-eight. This band is doubly-connected and is homeomorphic with the annulus $\frac{1}{2}<|z|<1$ in the $z$-plane. We can define a simply-connected region in the annulus by means of a spiral with an infinite number of turns which approach $|z|=1$. Consider, for instance, the spiral $z=\left(1-e^{-x}\right)(\cos x+i \sin x)$, $0<x<\infty$. That part of it which lies in the annulus defines such a simplyconnected region $W^{\prime}$, and this in turn defines in $S$ a simply-connected region 
$W$ bounded by a curve which traces an infinite succession of figure-eights; and approaches the figure-eight over the pair of circles $|w \pm i|=1$.

But then any surface $R^{\prime}$ of type II which contains $W$ must contain a simple closed curve consisting of the figure-eight over the circles $|w \pm i|=1$ traced a finite number of times. This is impossible, for such a curve would not divide $R^{\prime}$, whereas every simple closed curve on a surface of type II divides it.

The region $W$ can be mapped from the unit circle $|z|<1$ by a rational function of a mapping function. But since $W$ cannot be imbedded in a surface of type II, the rational function cannot be taken as a polynomial or a circular rational function. This completes our demonstration.

HARVARD UNIVERSITY,

Cambridge, Mass. 